\title{
Das neue Erwachsenenschutzrecht - wichtige Änderungen für die Psychiatrie
}

\section{René Bridler ${ }^{a}$,}

Jürg Gassmann ${ }^{b}$

a Dr. med., FMH Psychiatrie und Psychotherapie,

Ärztlicher Direktor

Sanatorium Kilchberg AG, Privatklinik für Psychiatrie und Psychotherapie

b Rechtsanwalt, ehemaliger Geschäftsleiter von Pro Mente Sana
* $\mathrm{nZGB}=$ Schweizerisches Zivilgesetzbuch (Erwachsenenschutz, Personenrecht und Kindesrecht), Änderung vom 19. Dezember 2009

\section{Korrespondenzen:}

Dr. med. René Bridler Sanatorium Kilchberg AG Alte Landstrasse 70 CH-8802 Kilchberg Tel. 0447164265 Fax 0447164343

r.bridler@sanatoriumkilchberg.ch

Jürg Gassmann

Zürcherstrasse 41 Gebäude 100 CH-8400 Winterhur Tel. 0522627043

info@gassmannlaw.ch
Am 19.12.2008 beschlossen beide Räte in der Schlussabstimmung mit grossem Mehr die Änderung des Schweizerischen Zivilgesetzbuches zum Erwachsenenschutz, das Referendum wurde nicht ergriffen. In den Detailberatungen zuvor waren kaum Differenzen zutage getreten und nur wenige Änderungen an der ursprünglichen Fassung des Bundesrates vorgenommen worden. Mit dem Beschluss wurde das fast hundertjährige Vormundschaftsrecht grundlegend erneuert. Im Gegensatz zur bisherigen Praxis muss das neue Recht zukünftig von interdisziplinären Fachbehörden angewandt werden, die anstelle der heute vielerorts üblichen Laienbehörden treten. Allerdings wird sich die Einführung des revidierten Zivilgesetzbuches (nZGB)* bis ins Jahr 2013 oder 2014 verzögern, da die Umsetzung der neuen Bestimmungen auf kantonaler Ebene einen erheblichen Verwaltungsaufwand erfordert.

Ein wichtiges Ziel der Revision war es, das Selbstbestimmungsrecht der Menschen zu fördern. Dazu wurde unter dem Titel «Die eigene Vorsorge» unter anderem die Patientenverfügung als neues Rechtsinstitut im Zivilgesetzbuch verankert. Mit der Patientenverfügung kann eine urteilsfähige Person im Voraus festlegen, welchen medizinischen Massnahmen sie im Falle einer Urteilsunfähigkeit zustimmt oder nicht zustimmt, oder eine natürliche Person bezeichnen, die im Fall ihrer Urteilsunfähigkeit in ihrem Namen über die vorgeschlagenen medizinischen Massnahmen entscheidet (Art. 370 nZGB). Mit der Revision wird zudem einheitlich geregelt, wem die Vertretungsbefugnis zusteht, wenn eine urteilsunfähige Person nicht mehr in der Lage ist, selbst über medizinische Massnahmen zu entscheiden. Fehlt eine rechtsverbindliche Patientenverfügung, regelt das Gesetz die Reihenfolge der Vertretungsberechtigung im Sinne einer klaren siebenstufigen Hierarchie (Art. 378 nZGB)

Die Neuregelung der Fürsorgerischen Freiheitsentziehung (FFE), die in Zukunft Fürsorgerische Unterbringung heisst (Art. 426-439 nZGB), beinhaltet einerseits Verfahrensvorschriften und beschränkt andererseits die ärztliche Einweisungskompetenz, wobei den Kantonen bei der Umsetzung wie bis anhin ein erheblicher Freiraum zugestanden wird. Die Voraussetzungen für die Einschränkung der Bewegungsfreiheit sind im Abschnitt über den Aufenthalt in Wohnoder Pflegeeinrichtungen geregelt und gelten sinngemäss auch bei fürsorgerischen Unterbringungen.

\section{Résumé}

Le nouveau droit de la protection des adultes, planifié d'entrer en vigueur en 2013 ou 2014, prévoit la possibilité des traitements médicaux ambulatoires obligatoires administrés contre la volonté de la personne concernée - faisant une réserve expresse en faveur du droit cantonal. Le nouveau droit concerne en première ligne la psychiatrie. Cet article éclaircit dans sa première partie paru dans ce numéro le cadre légal, les options thérapeutiques ainsi que des arguments courants en faveur de la prise en charge en ambulatoire de façon obligatoire. La deuxième partie, qui paraîtra dans le numéro prochain, donne une indication brève sur la littérature empirique concernant ce sujet et traite des arguments contre des mesures ambulatoires. Quelques propres conclusions critiques complètent cet article.*

* Wir danken Frau Dr. med. Heike Russmann für die Übersetzung

Nach Art. 383 nZGB darf die Bewegungsfreiheit einer urteilsunfähigen Person nur eingeschränkt werden, wenn weniger einschneidende Massnahmen nicht ausreichen oder von vornherein als ungenügend erscheinen (Verhältnismässigkeitsprinzip) und die Massnahme dazu dient,

- eine ernsthafte Gefahr für das Leben oder die körperliche Integrität der betroffenen Person oder Dritter abzuwenden oder

- eine schwerwiegende Störung des Gemeinschaftslebens zu beseitigen.

Art. 426 nZGB regelt die Fürsorgerische Unterbringung, wonach eine Person zur Behandlung oder Betreuung in einer geeigneten Einrichtung untergebracht werden darf, wenn

- sie an einer psychischen Störung oder an geistiger Behinderung leidet oder schwer verwahrlost ist und

- die nötige Behandlung oder Betreuung nicht anders erfolgen kann (Verhältnismässigkeitsprinzip). 


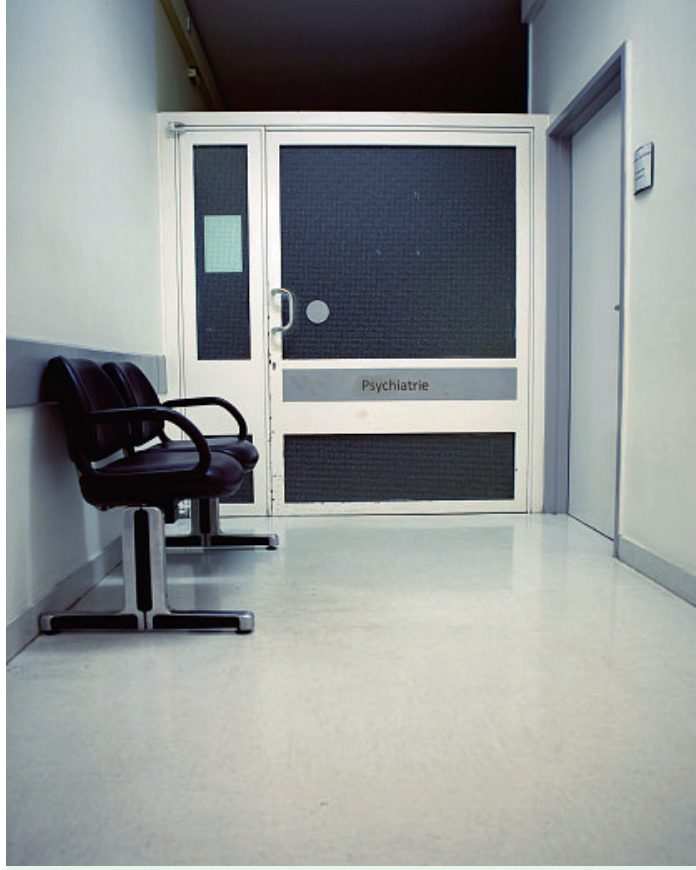

Das neue Erwachsenenschutzrecht sieht Möglichkeiten für ambulante medizinische Zwangsbehandlungen vor.

Im weiteren werden neu erstmals auf Bundesebene stationäre Zwangsbehandlungen abschliessend geregelt (Art. 434 nZGB). Demnach kann die Chefärztin oder der Chefarzt der Abteilung in einem Behandlungsplan vorgesehene medizinische Zwangsmassnahmen in Form einer Verfügung schriftlich anordnen, wenn kumulativ folgende Bedingungen erfüllt sind:

- Gefahr eines ernsthaften gesundheitlichen Schadens für die betroffene Person oder ernsthafte Gefährdung für die körperliche Integrität Dritter;

- Urteilsunfähigkeit der betroffenen Person bezüglich ihrer Behandlungsbedürftigkeit;

- Keine angemessene, weniger einschneidende Massnahme möglich (Verhältnismässigkeitsprinzip).

Von dieser Bestimmung nicht betroffen sind Notfallsituationen, in denen die zum Schutz der betroffenen Person oder Dritter unerlässlichen medizinischen Massnahmen auch in Zukunft unverzüglich ergriffen werden dürfen bzw. müssen. Sofern dabei der Institution der Behandlungswunsch der Person bekannt ist, ist auch in Notfallsituationen dieser Wille zu berücksichtigen (Art. 435 nZGB).

\section{Ambulante psychiatrische Zwangsbehand- lung im neuen Erwachsenenschutzrecht}

Der bundesrätliche Vorentwurf verzichtete ursprünglich auf die Möglichkeit von ambulanten Zwangsbehandlungen, unter anderem aus dem Grund, dass heute nur wenige Kantone eine entsprechende gesetzliche Regelung kennen. Dieser Verzicht wurde im
Laufe der Vernehmlassung indes stark kritisiert. In Aufnahme dieser Kritik wurde deshalb - mit einem ausdrücklichen Vorbehalt zugunsten des kantonalen Rechts - eine gesetzliche Grundlage geschaffen, um auch im ambulanten Setting Zwangsbehandlungen zu ermöglichen. Nach Art. 437 nZGB sind für die rechtliche Regelung der Nachbetreuung nach einem stationären Aufenthalt sowie der ambulanten Massnahmen die Kantone zuständig. Es bleibt ihnen überlassen, ob sie entsprechende gesetzliche Grundlagen schaffen oder auf die Möglichkeit ambulanter Zwangsmassnahmen verzichten wollen. Im Kanton Zürich wurde von der Justizdirektion diesbezüglich vor kurzem eine Vernehmlassung durchgeführt.

\section{Zeitpunkt der Anordnung von ambulanten $Z$ wangsbehandlungen}

Unter Berücksichtigung der neuen Gesetzgebung (Art. 437 nZGB) gibt es zwei Konstellationen, in denen in Zukunft ambulante psychiatrische Zwangsmassnahmen angeordnet werden können, sofern die kantonale Gesetzgebung solche vorsieht. Die beiden Situationen unterscheiden sich in erster Linie durch den Zeitpunkt ihrer Anordnung und weniger durch ihren Inhalt. Es handelt sich um die zwei folgenden Situationen:

- Auflage zur ambulanten psychiatrischen Nachbehandlung nach vorgängiger Fürsorgerischer Unterbringung (Art. 437 Ziff. 1 nZGB);

- Ambulante psychiatrische Behandlungsmassnahmen unabhängig von einer vorgängigen Fürsorgerischen Unterbringung (Art. 437 Ziff. 2 nZGB).

Bei der ersten Variante - im Falle einer mit Auflagen versehenen ambulanten Nachbetreuung - werden Zwangsmassnahmen bereits im Rahmen der Entlassungsvorbereitungen angeordnet, der Entscheid fällt noch während des Klinikaufenthaltes. Grundsätzlich kommen dafür Personen mit einer schweren psychischen Störung in Frage, die im stationären Setting nachweislich gut auf eine medikamentöse Therapie angesprochen haben, diese erfahrungsgemäss aber nach jedem Klinikaustritt beenden und eine ambulante psychiatrische Behandlung ablehnen oder rasch abbrechen. Dadurch geraten sie aufgrund der Vorgeschichte regelhaft in einen Krankheitszustand, der mit einer Eigen- oder Fremdgefährlichkeit oder auch mit einer schweren Verwahrlosung einhergeht und der schliesslich zu einer erneuten Fürsorgerischen Unterbringung mit Massnahmen wie Isolation oder Zwangsmedikation führt. Mit der Behandlungsauflage soll ein ausserstationäres, tragfähiges Therapiesetting geschaffen werden, um den Kreislauf eines ineffizienten Drehtüreffektes zu verlangsamen und schliesslich ganz zu unterbrechen.

Bei der zweiten Variante stehen die psychiatrischen Behandlungsmassnahmen nicht im Zusammenhang mit einem unmittelbar vorausgegangenen Klinikaufenthalt. Allerdings dürften bei der anvisier- 
ten Patientengruppe frühere fürsorgerische Unterbringungen eher die Regel als die Ausnahme sein. Hauptzweck ist es, die Betroffenen im ambulanten Setting zu halten oder neu in ein solches einzubinden und eine Klinikeinweisung nach Möglichkeit zu vermeiden. Die angeordnete Massnahme zielt also auf Personen mit der Fähigkeit, trotz einer schweren psychischen Störung in der Gemeinde zu leben, sofern sie die notwendige Unterstützung und Therapie erhalten. Im Falle einer ausbleibenden Behandlung kommt es bei ihnen aber regelmässig zu einer Verschlechterung des Gesundheitszustandes, der aufgrund früherer Erfahrungen zur Verneinung der Behandlungsbedürftigkeit und schliesslich zur schweren Eigen- oder Fremdgefährlichkeit mit der Notwendigkeit einer erneuten fürsorgerischen Unterbringung zur Behandlung führt. Analog zur Behandlungsanordnung nach einem Klinikaufenthalt geht es auch hier in erster Linie darum, den Kreislauf einer ungünstigen Spiralbewegung abzubremsen oder zu stoppen.

\section{Inhalt psychiatrischer Zwangsmassnahmen}

Entschliesst sich ein Kanton dazu, die rechtliche Grundlage für ambulante psychiatrische Zwangsbehandlungen zu schaffen, muss er auch die Kompetenzen und Behandlungsbefugnisse der mit der Durchführung betrauten Fachpersonen inhaltlich festlegen. Es sind drei Behandlungsmassnahmen denkbar, die sich durch eine schrittweise Zunahme ihres Zwangscharakters auszeichnen.

Die einfachste Massnahme besteht in einer ohne weitere Druckmittel versehenen Weisung, die angeordnete ambulante psychiatrische Behandlung wahrzunehmen. Die betroffene Person wird demnach verpflichtet, sich den auferlegten Therapien zu unterziehen, die Medikamente gemäss Verordnung einzunehmen und regelmässige Gesprächstermine wahrzunehmen. Sollte es zu einem Behandlungsabbruch mit einer Zustandsverschlechterung kommen, was eigentlich verhindert werden soll, muss auf das übliche Repertoire mit telefonischen Kontakten, schriftlichen Einladungen, Einbezug von Angehörigen und Arbeitgebern und aufsuchender Hilfe zurückgegriffen werden - je nach Möglichkeiten der Institution bzw. der Therapeuten. Darüber hinaus stehen keine weiteren Massnahmen zur Verfügung, es muss der natürliche Verlauf abgewartet und entsprechend gehandelt werden.

In Verschärfung der dargestellten Massnahme kommt, wie beispielsweise in den USA möglich [1], die Kompetenz hinzu, die Betroffenen einer Praxis oder einem sozialpsychiatrischen Ambulatorium polizeilich zuführen zu lassen, falls sie die Termine nicht wahrnehmen. Dieses Vorgehen gestattet dem Therapeuten, den persönlichen Kontakt zum Betroffenen und die regelmässige Beurteilung seines Gesundheitszustandes sicherzustellen und im Falle einer schweren Krankheitsepisode mit erheblicher Gefährdung eine erneute Fürsorgerische Unterbringung zu veranlassen.
Der schwerste Eingriff, wie z. B. Neuseeland ihn kennt [2], besteht in der Befugnis, im ambulanten Setting eine Zwangsmedikation mit Psychopharmaka durchzuführen. Der Patient kann also nicht nur hinsichtlich seines Krankheitszustandes klinisch beurteilt, sondern auch zur Einnahme eines Medikamentes gezwungen werden. Eine gewisse praktische Bedeutung würde diese Möglichkeit am ehesten bei der Verabreichung von Depotspritzen zur langfristigen medikamentösen Therapie von schweren Psychosen erlangen, während sich dagegen eine einmalige erzwungene Medikamenteneinnahme nur für Notfallsituationen und nicht zur langfristigen Behandlung eignet.

\section{Rechtliche Rahmenbedingungen}

Bei der Ausgestaltung der ambulanten Zwangsbehandlungen sind die Kantone an die rechtlichen Rahmenbedingungen gebunden, die sich aus dem übergeordneten Bundesrecht ergeben. Eine Zwangsbehandlung stellt in jedem Fall einen Eingriff in das verfassungsrechtlich geschützte Grundrecht der persönlichen Freiheit dar (Art. 10 Abs. 2 BV**). Ein solcher Eingriff setzt eine gesetzliche Grundlage voraus, muss durch ein öffentliches Interesse oder durch den Schutz von Grundrechten Dritter gerechtfertigt und überdies verhältnismässig sein (Art. $36 \mathrm{BV}$ ). Da eine Medikation ohne Zustimmung der betroffenen Person als schwerwiegende Einschränkung eines Grundrechts einzustufen ist, müssen die Voraussetzungen für den Eingriff im Gesetz selbst hinreichend klar geregelt sein (Art. 36 Abs. 1 BV). Mit dem Sinn und Zweck des Bundesrechts nicht vereinbar wäre unseres Erachtens eine kantonale Regelung, die eine Zwangsbehandlung auch in Situationen zuliesse, in denen sie im Rahmen einer fürsorgerischen Unterbringung

\section{Die Entscheidungsbefugnis einer vertretungsberechtigten Person muss Vorrang haben}

nicht statthaft wäre. Die Voraussetzungen für eine Behandlung ohne Zustimmung im stationären Rahmen (Art. 434 nZGB; s. oben) dürften deshalb als Mindeststandard auch bei ambulanten Zwangsbehandlungen einzuhalten sein.

Als unzulässig einzustufen wäre überdies eine kantonale Regelung, die für den Fall der Weigerung der betroffenen Person, ein bestimmtes Medikament einzunehmen oder sich sonst einer Therapie zu unterziehen, automatisch eine Klinikeinweisung als Sanktion bzw. Massnahme vorsähe. Einen solchen Automatismus schätzen wir als bundesrechtswidrig ein, da das Erwachsenenschutzrecht in Art. 426 nZGB (s. oben) abschliessend regelt, unter welchen Voraus- 
setzungen eine Fürsorgerische Unterbringung zulässig ist.

Die in einer rechtsgültig errichteten Patientenverfügung enthaltenen Anordnungen (Art. 370 nZGB; s. oben) müssten beim Entscheid über ambulante Zwangsbehandlungen ebenso berücksichtigt werden wie bei der Behandlungsplanung im Falle von fürsorgerischen Unterbringungen (Art. 433f. nZGB). Klärungsbedürftig ist die Frage, in welchem Verhältnis kantonale Regelungen über ambulante Zwangsmassnahmen zu den Bestimmungen des Erwachsenenschutzrechts über die Vertretung urteilsunfähiger Personen (Art. 378 ZGB) stehen. Die Entscheidungsbefugnis einer vertretungsberechtigten Person muss nach unserer Auffassung Vorrang haben. Eine ambulante Zwangsbehandlung nach kantonalem Recht kann hingegen dann zum Zuge kommen, wenn entweder keine vertretungsberechtigte Person vorhanden ist oder die Erwachsenenschutzbehörde dieser das Vertretungsrecht entzogen hat, weil die Interessen der urteilsunfähigen Person gefährdet oder nicht mehr gewahrt sind (Art. 381 nZGB).

\section{In Fällen, die «auf der Kippe» stehen, könnte eine ambulante Behandlungsmassnahme eine sinnvolle} Alternative zur Klinikeinweisung darstellen

3 Botschaft des Bundesrates zur Änderung des Schweizerischen Zivilgesetzbuches (Erwachsenenschutz, Personenrecht und Kindesrecht) vom 28. Juni 2006.

\section{Argumente zugunsten ambulanter psychiatrischer Zwangsmassnahmen}

In Befürwortung von ambulanten psychiatrischen Zwangsmassnahmen wird zumeist argumentiert, dass eine Fürsorgerische Unterbringung zur Behandlung stets die Ultima ratio darstellen muss. Nicht in allen Fällen ist indes eine stationäre Behandlung notwendig. Es gibt heikle Situationen im Verlauf psychischer Erkrankungen, welche auch in einem ambulanten Setting erfolgversprechend behandelt werden können [3]. Solche Situationen können beispielsweise infolge eines selbstgewählten Absetzens der Medikamente eintreten und im Sinne des Versagens einer adäquaten ambulanten Behandlung sowohl im Anschluss an eine Fürsorgerische Unterbringung als auch unabhängig davon auftreten. Als Folge der fehlenden Behandlung kann die betroffene Person erneut in einen derart schweren Krankheitszustand geraten, dass eine Fürsorgerische Unterbringung nach Art. 426 nZGB angezeigt ist. Sind die Unterbringungsvoraussetzungen nach Art. 426 nZGB erfüllt, hat die künftig zuständige Erwachsenenschutzbehörde gemäss bundesrätlicher Botschaft in ihrem Entscheid keinerlei Ermessensspielraum. Mit anderen Worten: Sie muss die betroffene Person zwingend mittels Fürsorgerischer Unterbringung in eine geeignete Einrichtung einweisen. Die gesetzliche Grundlage mag ein- deutig erscheinen, im klinischen Alltag besteht indes ein erheblicher Graubereich bei der Einschätzung des Gefährdungspotentials, und die resultierenden Entscheidungen («Klinik ja oder nein») erfolgen nicht mit wissenschaftlicher Präzision. Gerade in Fällen, die «auf der Kippe» stehen, könnte in Zukunft eine ambulante Behandlungsmassnahme eine sinnvolle Alternative zur Klinikeinweisung darstellen.

Es mag, wie oben erwähnt, durchaus Personen geben, die sich trotz schwerer psychischer Erkrankung einer angeordneten ambulanten Behandlung auch ohne polizeiliche Zuführung oder gar Zwangsmedikation unterziehen würden. Als Motiv kann dabei die Vermeidung einer Fürsorgerischen Unterbringung durchaus eine Rolle spielen. Im Rahmen der gesetzlich vorgeschriebenen, umfassenden Aufklärungspflicht müsste die betroffene Person unmissverständlich über diese drohende Alternative informiert werden. Es können aber auch krankheitsassoziierte Faktoren wie eine ausgeprägte Ambivalenz oder Wahngedanken den Ausschlag geben, die den Patienten davon abhalten, aus freien Stücken eine ambulante Behandlung in Anspruch zu nehmen. Aus psychiatrischer Sicht mag es daher wünschbar erscheinen, solche Patienten nicht sofort mangels Alternative in eine Klinik einweisen zu müssen. Stattdessen würde ihnen - wenngleich vorübergehend im Rahmen einer Massnahme - ein ambulantes Setting angeboten und dabei versucht, sie längerfristig in diesem halten zu können. Mit diesem Vorgehen würde ein Zeitfenster eröffnet mit der Möglichkeit, die Patienten durch persönliche Kontakte für eine ambulante Behandlung zu gewinnen und ein therapeutisches Setting aufzubauen. Grundsätzlich darf davon ausgegangen werden, dass eine ausserstationäre Behandlung von den Betroffenen und ihren Angehörigen trotz anfänglich höherer Belastung als erfolgreicher, weniger einschneidend und weniger stigmatisierend erlebt wird. Zudem würden ambulante Massnahmen dem ineffizienten, Ressourcen konsumierenden Kreislauf der «Drehtürpsychiatrie» mit Behandlungsabbrüchen, Zustandsverschlechterung und Zwangsmassnahmen entgegenwirken. Aus einer rechtsstaatlichen Perspektive kann es zudem als ein Gebot der Verhältnismässigkeit angesehen werden, schwer psychisch kranken Menschen, die im Rahmen akuter Episoden vorübergehend ihr Krankheits- und Behandlungsbewusstsein verlieren, als Alternative zur Klinikzwangseinweisung ein ausserstationäres Therapiesetting anzubieten.

Der zweite Teil dieses Beitrags mit dem Titel «Die Praxis der ambulanten Zwangsbehandlung in anderen Ländern» erscheint in der nächsten Ausgabe der Schweizerischen Ärztezeitung. Er bietet einen kurzen Überblick über die Literatur zum Thema und befasst sich mit Argumenten, die gegen eine ausserstationäre psychiatrische Zwangsbehandlung sprechen. 